\title{
Severe endometriosis may be considered in the differential diagnosis in young women presenting massive hemorrhagic ascites
}

\author{
Luiz Flavio Cordeiro Fernandes • Sergio Podgaec • \\ Guilherme Cutait Castro Cotti • Mauricio Simoes Abrao
}

Received: 29 April 2011 / Accepted: 8 June 2011 / Published online: 29 June 2011

(C) Springer-Verlag 2011

Keywords Ascites · Endometriosis · Ovarian cancer

\section{Introduction}

Endometriosis, the presence of endometrial tissue outside the uterine cavity, affects $10 \%$ of women in reproductive age and has immunological aspects conferring the disease an indubitable inflammatory trace [1]. Patients may present dysmenorrhea, chronic pelvic pain, dyspareunia, and infertility but rarely develops massive hemorrhagic ascites.

One of the components of peritoneal fluid in normal menstrual cycles is an ovarian exudate. The increase in vascular permeability along with some other factors as extremely high estrogen concentrations, and the presence of angiogenic factors, prostaglandins, histamines, and cytokines, may play an important role in the appearance of

L. F. Cordeiro Fernandes $\cdot$ S. Podgaec $\cdot$ M. S. Abrao

Endometriosis Clinic, Department of Obstetrics and Gynecology,

Hospital das Clínicas, School of Medicine,

University of São Paulo,

São Paulo, Brazil

G. C. Castro Cotti

Department of Colorectal Surgery, Hospital das Clínicas,

School of Medicine, University of São Paulo,

São Paulo, Brazil

S. Podgaec $(\bowtie)$

Rua João Moura 627, conjunto 23, Jardim América, CEP 05412-911 São Paulo, Brazil

e-mail: sergiopodgaec@me.com massive ascites. Inflammation increase the volume of exudation and, consequently, of the peritoneal fluid [2].

In the USA, cirrhosis represents $81 \%$ of the causes of ascites followed by cancer $(10 \%)$, heart failure $(3 \%)$, tuberculosis $(2 \%)$, dialysis $(1 \%)$, pancreatic disease $(1 \%)$, and other causes $(2 \%)$. Ovarian tumors, ovarian hyperstimulation syndrome, pelvic or peritoneal tuberculosis, and Meigs syndrome also can cause ascites. The correct and successful treatment depends on a precise diagnosis of its cause [3].

Most gynecologists are unaware that endometriosis can be related to massive ascites, leading to a misdiagnosis, especially when the symptoms includes loss of weight. To improve the knowledge of this kind of clinical case, we describe a patient that presented hemorrhagic ascites due to endometriosis $[2,4]$.

\section{Case presentation}

A 28-year-old nulliparous Afro-Brazilian woman sought medical care because of a progressive increase in abdominal girth and a weight loss of $5 \mathrm{~kg}$ over a period of 7 months. The patient did not present either fever, coughing, night sweats, digestive, or urinary complaints. She related regular menstrual periods with intense dysmenorrhea and deep dyspareunia. Physical examination revealed a distended, nontender abdomen with positive shifting dullness. No other clinical abnormalities were observed. Diagnostic hypothesis included peritoneal tuberculosis, hepatopathy, and ovarian tumor.

Laboratory tests were requested and results revealed hemoglobin level of $9.5 \mathrm{~g} / \mathrm{dL}$, normal liver biochemistry, normal tumor marker levels (CA 125, CEA, $\beta-\mathrm{HCG}$, and $\alpha$ fetoprotein), and negative serology for HIV and hepatitis B and 
C. PPD was negative. Chest X-ray was normal. An abdominal and pelvic ultrasound was performed. It showed no liver lesions, massive ascites, and no signs of deep endometriosis. No other abnormalities were found. It should be emphasized that spleen, pancreas, kidneys, uterus, and ovaries were normal.

An abdominal tap was performed and a "dark brown" bloody fluid was aspirated, revealing hemorrhagic ascites. Laboratory analysis revealed a cell count of $6,800 / \mathrm{mm}^{3}$, white cell count of $2,720 / \mathrm{mm}^{3}$ (44\% segmented, $46 \%$ lymphocytes, and $10 \%$ monocytes) with intense erythrophagocytosis, and an albumin level of $3.2 \mathrm{~g} / \mathrm{dL}$ leading to a serum-ascites albumin gradient (SAAG) of 1.2. Cytology for malignant cells and cultures were negative. Gram staining failed to detect any pathogen.

As diagnosis remained undefined, laparoscopy was performed and a total of 9.4 $\mathrm{L}$ of hemorrhagic fluid was drained from the peritoneal cavity (Fig. 1). Extensive adhesions were observed between the liver, transverse colon, sigmoid, uterus, and adnexa. Lesions suggestive of endometriosis were seen in the peritoneum, and the pouch of Douglas was completely obliterated. A biopsy was performed on a black lesion in the mesosigmoid. Histology confirmed the diagnosis of endometriosis due to fibrosis and extensive hemosiderin deposition, as well as the presence of endometrial glands and stroma, and no evidence of malignancy.

The patient's postoperative course was irreproachable, and the patient was discharged home 2 days after the surgery. She received 3 months of GnRH analogue treatment followed by continuous estrogen-progestin hormonal treatment. Ultrasound examination 3 and 6 months later revealed no ascites. One year later, the patient had no complaints and continued with no menses, using continuous combined birth control pills, once she has no fertility desires at the present moment.

\section{Discussion}

Ascites is usually related to hepatic disorders, abdominal-pelvic neoplasias, or infections such as peritoneal

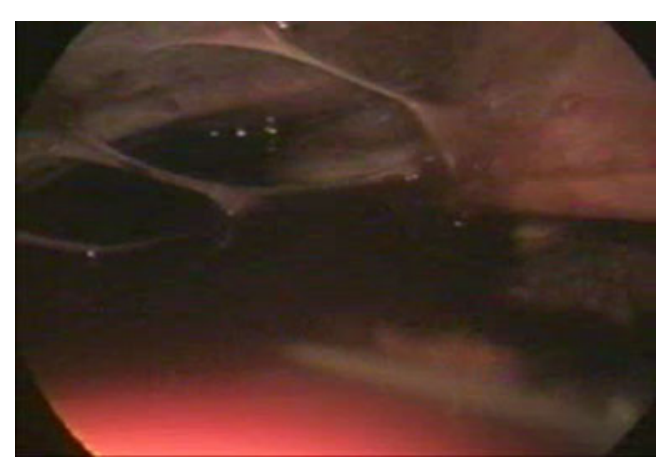

Fig. 1 Bloody massive ascites and multiple adhesions around the liver tuberculosis. Its correct diagnosis consists of a combination of a physical exam associated to an imaging test and to an adequate ascitic fluid analysis (abdominal paracentesis). As soon as the paracentesis is done, the gross appearance of the fluid can be extremely helpful in the differential diagnosis. The most common cause of ascites, cirrhosis, usually presents as a translucent yellow fluid. When it is infected, it appears normally turbid or cloudy. Bloody fluid usually is due to a "traumatic tap", but ascites is bloody in approximately $50 \%$ of patients with hepatocellular carcinoma and $22 \%$ of malignancy-related ascites at total. When it is brown, the patient may be deeply jaundice, what is easily seen in the physical exam, or has a ruptured gallbladder or perforated duodenal ulcer, both with a relevant history of a sudden pain [5]. The first three questions that we expect to answer when an abdominal tap is performed and we test the ascitic fluid are: (1) Is it infected?; (2) Is it related to a malignancy?; and (3) Does it show any signs of portal hypertension?

Although our patient showed a white cell count of $2,720 / \mathrm{mm}^{3}$ with $44 \%\left(1,197 / \mathrm{mm}^{3}\right)$ of segmented and intense erythrophagocytosis when the normal value would be a polymorphonuclear count $<250 / \mathrm{mm}^{3}$, we did not consider antibiotic treatment because of her history of 7 months of evolution with no fever, added to the appearance of the fluid. Decision proved correct when the culture showed no germs. Different from what we expected, the ascites of our patient had the traces of a transudate, with a SAAG of 1.2 $(\geq 1.1 \mathrm{~g} / \mathrm{dL}$ has an accuracy of $97 \%$ indicating portal hypertension), typical to hepatic origin, even though she had not any other feature going to this direction [2].

Endometriosis is a common, estrogen-dependent, benign, chronic inflammatory gynecological disease that is characterized by the presence of an endometrium-like tissue outside the uterine cavity, mainly on the pelvic peritoneum and ovaries. It affects $5 \%$ to $10 \%$ of women of reproductive age in the USA. The main clinical symptoms are dysmenorrhea, chronic pelvic pain, deep dyspareunia, and infertility [6].

The association of endometriosis and massive ascites is an extremely unusual condition. It was first related in 1958 and has been described in about 60 women up to date [4]. It constitutes an enormous dilemma for gynecologists owing to their scarcity and to their similarity to malignant ovarian neoplasias [7].

Women diagnosed with massive ascites related to endometriosis usually presents abdominal distention (71.4\%), weight loss $(60.3 \%)$, and abdominal pain $(52.4 \%)$. Ascites with endometriosis origin normally has the characteristics of an exudate, probably demonstrating a peritoneal etiology of the fluid due to peritoneal inflammation [8]. 
Those ascites are normally massive $(4,470 \pm 2,625 \mathrm{~L})$ and may be simultaneous to pleural effusion (42\%). It usually appears as dark brown or hemorrhagic, but without clots. Probably, the individual inflammatory reaction determines the clinical occurrence of ascites and/or pleural effusion. They also commonly come to a resolution after the excision of the endometriosis foci. These characteristics of the eventual concomitance of ascites and pleural effusion, additional to the normal resolution of the complication after the treatment and considering endometriosis as a benign condition, they all fit the criteria of Meigs syndrome, which is defined as the presence of ascites with hydrothorax in association with a benign ovarian tumor and the rapid resolution of fluid accumulation after tumor resection [9].

The definitive diagnosis is usually made during the surgical procedure, after excluding other clinical entities causing ascites. Once the correct diagnosis is made, the treatment is the same as that of endometriosis, consisting of supressing the ovarian function either surgically or with hormonal medications. Since the mean age of presentation is $31.9 \pm 8.8$ years and most of these patients are nulliparous, endocrine therapy may be preferred to surgical castration. Medical castration is normally less efficient than the surgical one and cannot prevent recurrence in some cases. That is why women receiving medical treatment need long follow-up with hormonal supression. The decision for the definitive surgical treatment is made based on several factors such as responsiveness to medical treatment and the desire for fertility [8].

During the follow-up, a question is raised when infertility is present and a controlled ovarian hyperstimulation $(\mathrm{COH})$ is needed. Will the symptons recur? Unfortunately, only some cases with ascites and endometriosis have been described and, as far as we are concerned, no data about this issue can be found. Although, taking into account that Benaglia et al. showed that in vitro fertilization and, consequently, $\mathrm{COH}$ did not seem to be associated to a higher rate of endometriosis recurrence, we expect the same findings regarding the recurrence rate of ascites. However, a multicenter prospective cohort study is warranted to address this specific question [10].

\section{Conclusion}

Gynecologists must be aware that endometriosis may cause massive ascites mimicking ovarian neoplasia. It should be considered in the differential diagnosis of hemorrhagic ascites in women at reprodutive age. The medical treatment is preferred than the radical surgical approach.

Conflicts of interest The authors report no conflicts of interest. The authors alone are responsible for the content and writing of the paper.

\section{References}

1. Bellelis P, Dias JA Jr, Podgaec S, Gonzales M, Baracat EC, Abrao MS (2010) Epidemiological and clinical aspects of pelvic endometriosis: series of cases. Rev Assoc Med Bras 56(4):467471

2. Ussia A, Betsas G, Corona R, De Cicco C, Koninckx PR (2008) Pathophysiology of cyclic hemorrhagic ascites and endometriosis. J Minim Invasive Gynecol 15(6):677-681. doi:10.1016/j. jmig.2008.08.012

3. Runyon BA, Montano AA, Akriviadis EA, Antillon MR, Irving MA, McHutchison JG (1992) The serum-ascites albumin gradient is superior to the exudate-transudate concept in the differential diagnosis of ascites. Ann Intern Med 117(3):215-220

4. Gungor T, Kanat-Pektas M, Ozat M, Zayifoglu Karaca M (2011) A systematic review: endometriosis presenting with ascites. Arch Gynecol Obstet 283(3):513-518. doi:10.1007/s00404-010-1664-1

5. Andrade DR Jr, Galvao FH, Santos SA, Andrade DR (2009) Ascite-state of the art based on evidences. Rev Assoc Med Bras 55(4):489-496

6. Bulun SE (2009) Endometriosis. N Engl J Med 360(3):268-279. doi:10.1056/NEJMra0804690

7. Goumenou A, Matalliotakis I, Mahutte N, Koumantakis E (2006) Endometriosis mimicking advanced ovarian cancer. Fertil Steril 86 (1):219 e223-215. doi:10.1016/j.fertnstert.2005.12.044

8. Sait KH (2008) Massive ascites as a presentation in a young woman with endometriosis: a case report. Fertil Steril 90(5):2015 e2017-2019. doi:10.1016/j.fertnstert.2008.07.021

9. Lurie S (2000) Meigs' syndrome: the history of the eponym. Eur J Obstet Gynecol Reprod Biol 92(2):199-204

10. Benaglia L, Somigliana E, Vercellini P, Benedetti F, Iemmello R, Vighi V, Santi G, Ragni G (2010) The impact of IVF procedures on endometriosis recurrence. Eur J Obstet Gynecol Reprod Biol 148(1):49-52. doi:10.1016/j.ejogrb.2009.09.007 